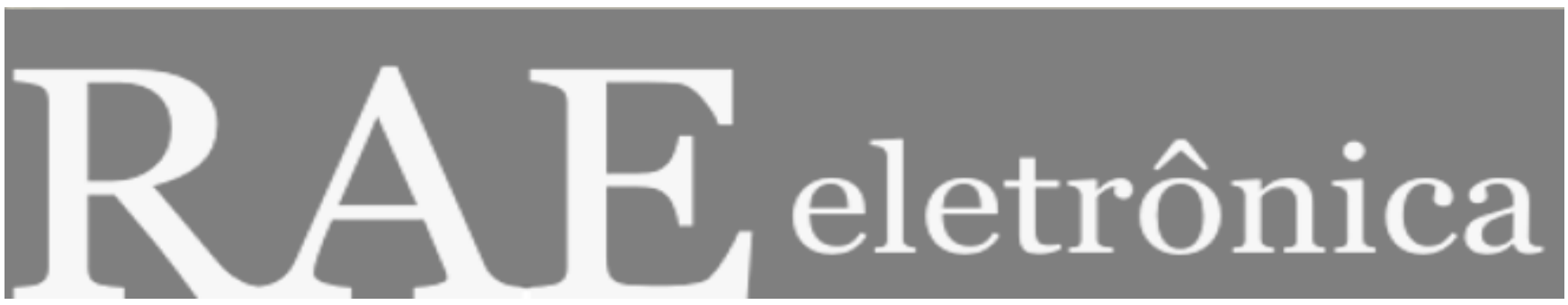

\title{
SILÊNCIO E OMISSÃO: ASPECTOS DA CULTURA BRASILEIRA NAS ORGANIZAÇÕES
}

Por

Francisco Giovanni David Vieira

João Marcelo Crubellate

Ilse Gomes Silva

Wânia Rezende Silva

RAE-eletrônica, Volume 1, Número 1, jan-jun/2002.

http://www.rae.com.br/eletronica/index.cfm?FuseAction=Artigo $\&$ ID $=1017 \&$ Secao=ORGANIZA\&Volume $=1 \& N u m e r o=1$ $\& A n o=2002$

CCopyright, 2002, RAE-eletrônica. Todos os direitos, inclusive de tradução, são reservados. É permitido citar parte de artigos sem autorização prévia desde que seja identificada a fonte. A reprodução total de artigos é proibida. Os artigos só devem ser usados para uso pessoal e nãocomercial. Em caso de dúvidas, consulte a redação: redacao@rae.com.br.

A RAE-eletrônica é a revista on-line da FGV-EAESP, totalmente aberta e criada com o objetivo de agilizar a veiculação de trabalhos inéditos. Lançada em janeiro de 2002, com perfil acadêmico, é dedicada a professores, pesquisadores e estudantes. Para mais informações consulte o site www.rae.com.br/eletronica.

RAE-eletrônica

ISSN 1676-5648

(C)2002 Editora: Fundação Getulio Vargas - Escola de Administração

de Empresas de São Paulo.

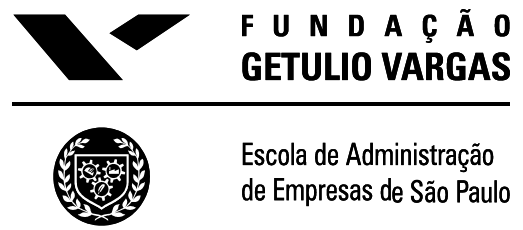




\section{SILÊNCIO E OMISSÃO: ASPECTOS DA CULTURA BRASILEIRA NAS ORGANIZAÇÕES}

\section{Francisco Giovanni David Vieira}

Bacharel em Administração de Empresas pela UFPB, Mestre em Administração pela UFLA, Doutorando em Ciências Sociais pela PUC-SP, Professor-Assistente do Departamento de Administração da UEM e Co-Editor da Revista Interdisciplinar de Marketing.

E-mail: fgdvieira@wnet.com.br

\section{João Marcelo Crubellate}

Bacharel em Administração de Empresas pela UEM, Mestre em Administração pela UFPR, Doutorando em Administração pela FGV-EAESP e Professor-Assistente do Departamento de Administração da UEM.

E-mail: jmcrubellate@uem.br

\section{Ilse Gomes Silva}

Bacharel em Enfermagem pela UEMA, Mestre e Doutoranda em Ciências Sociais pela PUC-SP e Professora-Assistente do Departamento de Enfermagem da UEMA.

E-mail: $\underline{\text { ilsegs@sti.com.br }}$

\section{Wânia Rezende Silva}

Bacharel em Ciências Sociais pela UFMG, Mestre em Administração pela UFLA, Doutora em Ciências Sociais pela PUC-SP e Professora Adjunta do Departamento de Ciências Sociais da UEM.

E-mail: waniasilva@yahoo.com

\section{RESUMO}

O artigo analisa o silêncio e a omissão - aspectos da cultura brasileira - presentes nas organizações empresariais e estatais, que não só moldam a maneira como tais organizações se relacionam entre si e com o mercado, como também influenciam a própria formação e desenvolvimento dos processos e medidas administrativas dessas organizações. O pano de fundo para tal análise é a falsificação de remédios no Brasil, e a sua referência empírica são as organizações a ela vinculadas.

\section{ABSTRACT}

The paper analyzes the silence and the omission - aspects of the Brazilian culture -presents in the private and public organizations, that not only molds the way as such organizations link to each other and with the market, as well as influence the formation and development of the administrative processes of those organizations. The background for the analysis is the falsification of medicines in Brazil, and the organizations linked to it are its empiric reference.

\section{PALAVRAS-CHAVE}

Cultura organizacional, cultura empresarial brasileira, empresa privada e Estado, remédios.

\section{KEY WORDS}

Culture organizational, Brazilian managerial culture, private company and State, medicines 


\section{INTRODUÇÃO}

Em 1998 veio à tona no Brasil uma situação de largas proporções, que expôs procedimentos gerenciais significativos para a análise e compreensão de aspectos da cultura brasileira nas organizações. Tal situação diz respeito à falsificação de remédios que, em escândalos e mais escândalos subsequentes, e em várias regiões, deixou a população alarmada e jogou o país num furacão de medos sobre remédios falsificados e adulterados.

No centro desse furacão estava um medicamento contraceptivo popular e de uso generalizado no país. Esse medicamento não possibilitou a contracepção e várias mulheres que dele fizeram uso, engravidaram. A empresa responsável pela fabricação do produto, uma multinacional que, à época, era a sétima empresa farmacêutica do país em unidades vendidas e a décima segunda em faturamento do ramo, foi informada do acontecimento, tanto por parte das consumidoras, quanto por parte dos revendedores, e não tomou providências no sentido de esclarecer a situação ou resolver o problema pelo menos por um período de trinta dias, até o caso ser noticiado pela principal emissora de televisão do país.

As apurações quanto à responsabilidade civil e responsabilidade criminal, revelaram fatos ligados a produção de placebos (mistura de farinha de trigo, amido e outros materiais inertes) para testes de embalagens, negligência no acondicionamento e manejo do material resultante do teste de embalagens, roubos internos e venda ao mercado através da conivência de distribuidoras de produtos farmacêuticos e de farmácias - empresas varejistas. Como pedras de um dominó que vão caindo uma após a outra, ou como efeitos colaterais desse caso inicial, foram descobertas várias falsificações e adulterações de medicamentos em diversas regiões do Brasil. O padrão da falsificação de remédios que se observou a seguir, entretanto, diferentemente do que poderia ser considerado um acidente de percurso, revelou a existência assustadora de uma série de problemas e, sobretudo, de uma rotina e de uma capacidade instalada e concebida para a falsificação (Quadro 1), como se nada a ameaçasse, inclusive o próprio Estado Brasileiro através da sua vigilância sanitária.

- A população brasileira é a quarta maior consumidora de medicamentos do mundo. Perde apenas para os americanos, franceses e alemães;

- Há no país 55000 farmácias. Segundo a OMS o número ideal seria de, no máximo, 25000;

- O elo entre a indústria e os mercados varejistas no setor farmacêutico é o conjunto das distribuidoras. De cada 100 produtos farmacêuticos, 85 são comercializados pelas distribuidoras;

- Os medicamentos representam, em valores, o maior volume de cargas roubadas nos Estados de São Paulo e Rio de Janeiro, que estão entre os principais centros consumidores. As cargas de medicamentos representaram $36 \%$ do valor total roubado nos maiores assaltos.

- No Brasil de cada duas pessoas, uma é adepta da automedicação;

- Há leis mas não há fiscalização. De cada três remédios vendidos no país apenas um é receitado por médico;

- Os brasileiros são supermedicados. Um cidadão sadio consome, nos países desenvolvidos, em média, três caixas de remédio por ano. Aqui o mesmo cidadão sadio consome onze;

- Metade dos medicamentos prescritos é desnecessária. A maioria é contra gripe.

Fonte: Ministério da Saúde, Abifarma, e Antônio Carlos Zanini, Consultor da OMS, apud Veja (1998).

Quadro 1 - Alguns dos motivos que facilitam o comércio de remédios ilegais no Brasil 
Essa constatação refletia-se em dados relativos ao setor. Segundo levantamento de Siqueira (1998), já naquela época o faturamento do setor farmacêutico no Brasil chegava a US\$ 11 bilhões por ano. Os números das falsificações, portanto, são demasiadamente eloqüentes ao observar-se que de acordo com dados da Organização Mundial da Saúde (OMS), apresentados por Pastore (1998), cerca de $10 \%$ de todo esse faturamento está sendo desviado para a indústria da fraude. Ainda segundo esses mesmos dados da OMS, são vendidos 20 medicamentos falsos em cada lote de 100. Tais evidências numéricas são recrudescidas ao levar-se em consideração declarações e reconhecimento do presidente da Associação Brasileira da Indústria Farmacêutica - Abifarma, prestados a Edward e Cardoso (1998), de que os Laboratórios geralmente exageram na publicidade e muitas vezes preferem calar-se a denunciar fraudes em seus produtos.

O aparato estatal vinculado à indústria farmacêutica, por sua vez, dispunha, à época, de apenas 20 técnicos para registrar todo e qualquer tipo de medicamento, e 1.400 inspetores para controlar cerca de 400 laboratórios, 7.000 distribuidoras de remédios e 55.000 farmácias (Ministério da Saúde e Abifarma apud Pastore, 1998). Esse aspecto da estrutura administrativa estatal é agravado pelas próprias políticas públicas, seja do ponto de vista da ação de fiscalização ou seja da legislação em vigor. Isso, sem levar em consideração a definição do que sejam as prioridades governamentais em relação à dotação orçamentária para assuntos de saúde pública, incluindo-se tratamento e prevenção de doenças, como mostram dados divulgados pelo Ministério da Saúde, em que se notifica que os gastos com a saúde caíram 12,4\% em relação ao PIB entre os anos de 1994 a 1998.

Diante de tais evidências relacionadas às empresas privadas e ao Estado Brasileiro frente à falsificação de remédios no Brasil, observa-se a existência de silêncio, por um lado, e omissão, por outro. O pressuposto fundamental assumido neste ensaio é o de que esse silêncio e essa omissão são aspectos da cultura brasileira que estão presentes nas organizações empresarias e estatais, os quais não só têm moldado a maneira como tais organizações se relacionam entre si e com o mercado, como também têm influenciado a própria formação e desenvolvimento dos processos e medidas administrativas dessas organizações. A análise dessa questão, particularmente sob a óptica da cultura organizacional, é o centro do ensaio. Ele insere-se no esforço sugerido por Prestes Motta e Caldas (1997) de entender melhor como a cultura brasileira é expressa e revelada em nossas organizações. Para tanto, divide-se em quatro momentos: o primeiro, definido como o quadro teórico, apresenta os fundamentos da análise aqui realizada, os quais estão alicerçados na cultura brasileira e no conceito de cultura organizacional; o segundo enfoca o aspecto do silêncio das empresas, abordando mais diretamente o caso que deflagrou a constatação empírica da falsificação de remédios no Brasil; o terceiro compreende uma visão geral da omissão do Estado Brasileiro sobre a questão da saúde; o quarto momento consiste da realização de considerações finais a respeito da análise aqui empreendida.

\section{QUADRO TEÓRICO}

\section{Cultura nas ciências sociais}

Segundo Cuche (1999), a noção de cultura é inerente à reflexão das ciências sociais. Tal noção parece fornecer a resposta mais satisfatória à questão da diferença entre os povos, dado que a sua consolidação enquanto conceito científico implicou no estudo de sua evolução histórica, a qual não ocorreu sem que sempre tenha estado presente divergência semântica sobre a definição da palavra, desacordos sociais (lutas sociais) e até nacionais, como o debate franco alemão sobre cultura e civilização. 
Além disso, ainda de acordo com Cuche (1999), a invenção do conceito científico de cultura trilhou um longo caminho. Em linhas gerais, o seu percurso dentro das ciências sociais deu-se conforme os seguintes momentos: sistematização inicial com Tylor e a concepção universalista da cultura; Franz Boas e a concepção particularista de cultura; Malinowsky e a análise funcionalista da cultura; o advento da antropologia cultural; Lévi-Strauss e a análise estrutural da cultura; a noção de interacionismo cultural; a discussão sobre subcultura; o conceito de aculturação e culturalismo; a idéia de cultura dominante e cultura dominada; a especificação de cultura popular, cultura de massa e cultura de classe; a discussão sobre cultura e identidade, bem como sobre cultura política.

Todo esse percurso tem como principal força motriz, a concepção e constatação, a um só tempo, de que o homem é essencialmente um ser de cultura. De onde é a cultura que torna possível a transformação da natureza e que faz com que, portanto, as populações se diferenciam pelas suas escolhas culturais, invenções e resoluções diferentes de problemas.

É nessa perspectiva que, conforme Laraia (1997), para se compreender o mundo e o próprio comportamento humano, é preciso levar em consideração as diferentes concepções de mundo e sistemas de valores. Dessa forma, para as ciências sociais, a noção de cultura compreendida em seu sentido vasto, remete aos modos de vida e de pensamento. Assim, ela é hoje bastante aceita e apesar da existência de certas ambigüidades, ela oferece a possibilidade de conceber a unidade do homem na diversidade de seus modos de vida e de crença, enfatizando a unidade ou a diversidade (Cuche, 1999).

\section{CULTURA BRASILEIRA E ORGANIZACIONAL}

É de conhecimento geral o caráter fragmentário e multifacetado de nossa cultura, resultado das raízes multiraciais do povo brasileiro. Essas características tornam mais problemático do que já é em relação a outras culturas mais sedimentadas, buscar-se aspectos distintivos e com suficiente grau de compartilhamento social que permitam identificar alguma base cultural no país. Tais traços, contudo, existem, ou seria o caso de ser mesmo sem qualquer sentido falar-se em cultura brasileira. Os trabalhos realizados por vários estudiosos e pensadores, como Freire (1963), Caio Prado (1989) e Darcy Ribeiro (1995), entre outros, são grandes exemplos e referências teóricas da mesma.

Um traço cultural, entretanto, da sociedade brasileira, destacado no presente ensaio e com evidentes impactos sobre a nossa organização social, inclusive em termos econômicos, diz respeito à dificuldade em se constituir e consolidar instituições. Segundo DaMatta (1983), no Brasil as agremiações, associações ou quaisquer outros tipos de grupos são constituídos de forma a abranger a maior quantidade de diferentes representações sociais, faltando-lhes uma definição de fronteiras internas e externas para o agrupamento. Como conseqüência, tais grupos terminam representando a todos e a ninguém ao mesmo tempo, exatamente porque não conseguem se articular em torno de interesses bem definidos. Vê-se, portanto, impedida "(...) a formação de grupos de interesse politicamente representativos e, consequentemente, politicamente poderosos” (DaMatta, 1983:106).

Tal dificuldade não é encontrada, apenas como exemplo para marcar a análise em termos de comparação com outros contextos culturais, em países com marcante herança do protestantismo ascético. Como analisa Souza (1998), a impessoalidade das relações sociais nessas culturas, resultado principalmente da necessidade de subjugar as emoções ao controle racional, conduz à predominância de relações calcadas no interesse comum, em que a base não é afetiva, mas racional em função de interesses. 
No Brasil, de modo diferente, há uma predominância do conteúdo emocional na cultura de um modo geral. Isso inspirou Buarque de Holanda (1975) a cunhar o conceito de "homem cordial" como típico do indivíduo brasileiro, que se manifesta pela sua aversão a qualquer tipo de ritualismos e que somente aceita relações sociais com base pessoal. Para Souza (1998:106), esse homem cordial é a oposição simétrica do protestante ascético, sendo caracterizado por um individualismo personalista, busca de prazeres imediatos e descaso por ideais comunitários e de longo prazo.

Essas características têm desdobramentos na nossa vida religiosa e social, na nossa linguagem e também nas nossas relações comerciais. Não há como obscurecer, nesse sentido, que elas causem impacto direto sobre a vida organizacional, seja através da passividade e a aceitação do autoritarismo, seja através de paternalismo nas relações, onde a troca de pequenos e imediatos favores desenvolve uma dependência moral para com o outro, dissuadindo o questionamento e o rompimento da relação (Freitas, 1997; Prestes Motta, 1999). O que queremos fazer perceber é que tais aspectos da cultura brasileira fornecem subsídios para diferentes ações, tanto das organizações econômicas, mesmo que oriundas de outros contextos culturais, quanto do próprio governo, por parte de suas agências, no seu relacionamento com essas organizações e com a sociedade civil mais ampla. Essa percepção, do ponto de vista da teoria das organizações, encontra amparo em estudos como os de Smircich (1983), Hofstede (1991), Martin (1992) e Morgan (1996), por exemplo

\section{O SILÊNCIO DAS EMPRESAS}

A reação esboçada pela indústria farmacêutica cujo caso iniciou a revelação da magnitude da falsificação e adulteração de medicamentos no Brasil foi, no mínimo, um exemplo típico de comunicação empresarial mal sucedida. Tendo conhecimento de reclamações e problemas decorrentes do uso de um medicamento produzido por ela, a empresa resolveu ficar em silêncio e não tomar nenhuma providência. Com o passar do tempo e o surgimento de novos casos, percebeu-se que esse silêncio era a regra e não a exceção da indústria farmacêutica perante casos semelhantes.

Após produzir duas toneladas de placebos, em que $1.200 \mathrm{Kg}$ - o correspondente a 650 mil cartelas de anticoncepcionais - foram usados nos testes de embalagem, a empresa demorou 30 dias para perceber os problemas oriundos do desvio dos mesmos e fazer denúncias à Polícia Civil. Não foram suficientes os telefonemas e as cartas recebidas pelo SAC - Serviço de Atendimento ao Consumidor da mesma. Pareceram não ser suficientes também, as disposições legais e jurídicas contidas no Código de Defesa do Consumidor, o que a rigor foi confirmado pela série de fatos posteriores.

Embora, como afirme Penteado Filho (1990) e sugira Richers (1996), não seja fácil praticar marketing no Brasil e, por outro lado, o país seja considerado um terreno fértil para falsificação e adulteração de remédios, o papel desempenhado pelos SACs no âmbito organizacional e no processo decisório empresarial da indústria farmacêutica revelou-se contrário às características de funcionamento para as quais são implantados pelas empresas. Isso pode ser observado, por exemplo, através de uma comparação entre as ações apresentadas pelo uso dos SACs das empresas farmacêuticas e aquilo que é descrito como resultados do estudo realizado por Isnard (1997) em várias empresas de bens de consumo no país.

De modo complementar, estratégias de marketing que fossem compreendidas como marketing de defesa, priorizando o desenvolvimento de uma relação efetiva de solução de problemas dos consumidores, também não foi percebida. A relação foi de desconfiança e descrédito para com os consumidores e até descaso com a própria legislação. Essa última questão desperta curiosidade no que 
se refere à percepção da cultura do país e remete à idéia de uma conduta administrativa bastante distinta em empresas de um mesmo grupo. O que confirma essa observação é a revelação de Bitencourt (1998) de que existem suspeitas dentro da própria Comunidade Econômica Européia que as empresas européias se instalam nos países em desenvolvimento também pelo fato de neles encontrarem leis mais brandas para a concretização de suas atividades. Isso implica em uma clara negação da existência de uma predisposição em usar integralmente um modelo de marketing de defesa para se lidar com reações adversas, como, por exemplo, o modelo sugerido por Santos (1997). Implica, também, em descartar uma nítida possibilidade de aperfeiçoar as relações intra e extra-empresa (Morrison e Milliken, 2000).

Ao adotar um procedimento desse tipo, ou seja, ao ficar em silêncio, a indústria farmacêutica em toda a sua extensão optou por ficar "malandramente em cima do muro". Segundo DaMatta (1998), esse é um padrão de comportamento cultural que parece ser algo honesto, necessário e prático no caso do sistema social brasileiro. Talvez, assim, a indústria farmacêutica tenha tentado dar um "jeitinho" ao avesso, pois não tomou a iniciativa de tentar resolver a situação. Tratou-se de um "jeitinho" contrário aquilo que Barbosa (1992) descreve em sua tese como um dos mais instigantes procedimentos sociais do país, o chamado "jeitinho brasileiro". De qualquer forma, observa-se que nesse episódio a indústria farmacêutica desprezou o seu modelo de origem, estrangeiro, e adotou um modelo de administração e gerência baseado na cultura brasileira, o que implica em uma espécie de antropofagia organizacional em sentido inverso (Faria, Carvalho e Collares dos Santos, 2001).

\section{OMISSÃO DO ESTADO}

Na última década, a reforma do Estado adquiriu um caráter central na agenda política brasileira. Procurou-se adequar as políticas estatais aos objetivos de uma economia globalizada. Nesse sentido, as medidas para inserir o país na dinâmica da globalização foram a desregulamentação da economia, a flexibilização da legislação, a diminuição dos gastos públicos, a privatização das estatais, e a abertura do mercado aos investimentos transnacionais, dentre outras medidas. O modelo de Estado SocialLiberal, proposto então, teve como objetivo estimular e preparar as empresas e o país para a competição generalizada através da privatização, da publicização e da terceirização. Nesse modelo, distinguem-se as atividades exclusivas do Estado, dos serviços sociais e científicos, e da produção de bens e serviços para o mercado.

As atividades exclusivas do Estado ficaram circunscritas as funções de legislar, regular, julgar, policiar, fiscalizar e definir políticas. O controle social, entretanto, previsto pelo governo, passou a ser informal, eventual e baseado em critérios de eficiência e de apresentação de resultados.

Foi nesse contexto que se inseriram as denúncias de falsificação de medicamentos. Tais denúncias, ao alcançarem visibilidade na mídia, evidenciaram a debilidade da fiscalização no processo de fabricação, distribuição e venda dos medicamentos. A função da vigilância sanitária foi amplamente questionada em "estabelecer critérios, parâmetros e métodos para o controle da qualidade sanitária de produtos, substâncias e serviços de consumo e uso humano” (Lei 8.080/90 - Art. 16; inciso VIII).

A política de cortes nos gastos públicos e o processo de privatização na saúde fizeram aflorar uma realidade que ao longo dos anos não foi bem observada. Embora a saúde tenha sido reconhecida como direito de todos e dever do Estado em 1988 com a Constituição Federal, um dos serviços previstos, relativo à vigilância sanitária, foi prejudicado com a falta de recursos, e não teve condições estruturais e políticas para fiscalizar e controlar a produção e a comercialização de medicamentos, entre outras atividades de sua competência. 
O Estado, portanto, tem favorecido ao longo dos anos uma política cuja característica remete à idéia de sua omissão para com procedimentos que lesam os consumidores nos seus direitos básicos. Essa percepção é ratificada perante o anúncio da proposta de privatização da vigilância sanitária através do ente jurídico das Organizações Sociais, com autonomia e vinculada ao Ministério da Saúde por meio de um contrato de gestão.

\section{CONSIDERAÇões FINAIS}

Entender ações organizacionais a partir de uma perspectiva cultural, implica analisá-las para além dos aspectos formais e objetivos, certamente presentes nos processos que desencadeiam tais ações. As estratégias formais, as limitações estruturais e financeiras, além de dimensões internas e externas são importantes fatores que delimitam as possibilidades que se oferecem a qualquer organização em face das inúmeras e diversas circunstâncias do cotidiano empresarial. Contudo, abordagens que se limitem a tais dimensões podem ser induzidas pela expectativa de uma extrema racionalidade e linearidade nos processos organizacionais com resultados incompletos, quando não ilusórios. Nesse sentido, a perspectiva cultural significa, entre outras possibilidades, resgatar aspectos subjetivos que se mostrem significativos na formulação das respostas oferecidas pela organização (ou, mais apropriadamente, por seus membros) para os problemas operacionais e institucionais com que se defronta.

A cultura e os aspectos a ela vinculados, como o silêncio ou omissão, são resultados de construções. As identidades deles resultantes, segundo observações de estudiosos como Hall (1998), não são coisas com as quais nós nascemos, mas são formadas e reformadas no interior da representação. Assim, a relação entre cultura brasileira e cultura organizacional no que diz respeito às ações empresariais e estatais frente à falsificação de remédios no Brasil evidenciam exatamente um processo de construção e reconstrução simbólica, como foi evidenciado ao longo deste ensaio.

Por um lado percebe-se uma tendência histórica do Estado em ausentar-se de questões consideradas fundamentais, o que remonta ao Brasil colônia (Schwartz, 1999; DaMatta, 1983). Por outro, percebe-se que empresas no momento de estabelecerem relações de mercado com os consumidores desenvolvem uma espécie de apreensão funcional de valores e procedimentos sociais intrínsecos à cultura brasileira. É, nesse último caso, a não existência de uma identidade autêntica, mas sim de uma pluralidade de identidades e faces culturais, as quais, muitas vezes, inserem-se em um esforço de comunicação para além de aparentes paradoxos estruturais e culturais (Mooij, 1998; Ortiz, 1986).

Uma luz no fim do túnel, não obstante, pode ser ilustrada por meio da observação de Pinsky e Eluf (1993), em meio a comentários sobre vários aspectos do cotidiano brasileiro que atentam contra os direitos do cidadão: é questionável essa suposta índole de que o brasileiro seja preguiçoso e de que as leis existem no Brasil exatamente para não serem cumpridas. Ou será que sim, desde que seja por quem de "direito" ou por quem tenha "condições" de não cumpri-las? Como pôde ser visto neste ensaio, a análise da cultura brasileira, vinculada à cultura organizacional de empresas que aqui desenvolvem suas atividades, contribui para entender tal questão, sobretudo quando características como a aversão ao conflito e a distância do poder facilitam a disseminação de crenças quanto a um determinado caráter nacional e permitem a institucionalização de processos administrativos. A abordagem de tais aspectos, embora aqui realizada nos limites de um ensaio teórico, contribui no sentido de melhor entender como a cultura brasileira se expressa e é revelada nas organizações brasileiras, ao mesmo tempo em que molda os seus processos administrativos.

\section{Artigo recebido em 23.03.2003. Aprovado em 07.11.2001.}




\section{REFERÊNCIAS BIBLIOGRÁFICAS}

BARBOSA, Lívia. O jeitinho brasileiro: a arte de ser mais igual que os outros. Rio de Janeiro: Campus, 1992.

BITENCOURT, Silvia. Imprensa alemã fala de desgaste de filial. Folha de São Paulo, São Paulo, 09 jul. 1998. $3^{\circ}$ Caderno - Cotidiano, p.3.

CONSTITUIÇÃO FEDERAL DO BRASIL - 1988. Brasília: Tecnopriah, 1988.

BUARQUE DE HOLANDA, Sérgio. Raízes do Brasil. $8^{\text {a }}$ ed. Rio de Janeiro: José Olympio Editora, 1975.

CUCHE, Denys. A noção de cultura nas ciências sociais. Bauru: EDUSC, 1999.

DaMATTA, Roberto. Carnavais, malandros e heróis: para uma sociologia do dilema brasileiro. $4^{\mathrm{a}}$ ed. Rio de Janeiro: Zahar, 1983.

DaMATTA, Roberto. O que faz o brasil, Brasil? Rio de Janeiro: Rocco, 1998.

EDWARD, José e CARDOSO, Rodrigo. As novas vítimas. Veja, São Paulo, n. 1555, 15 jul. 1998. p.44-46.

FARIA, Alexandre; CARVALHO, José L. F. dos S. e COLlARES DOS SANTOS, Lyla. Antropofagia, antropofagia organizacional e estudos tribais: em busca da conciliação dialética de arte, cultura e management no Brasil. In: XXV ENCONTRO DA ASSOCIAÇÃO NACIONAL DOS PROGRAMAS DE PÓS-GRADUAÇÃO EM ADMINISTRAÇÃO - ENANPAD. (2001: Campinas). Anais ... Ensino e Pesquisa em Administração. Rio de Janeiro: Associação Nacional de Programas de Pós-Graduação em Administração, 2001. p. 111. (Texto Integral em CD-ROM dos Anais do $25^{\circ}$ ENANPAD).

FREIRE, Gilberto. Casa grande e senzala. $12^{\mathrm{a}}$ ed. Brasília: UNB, 1963.

FREITAS, Alexandre B. Traços brasileiros para uma análise organizacional. In: PRESTES MOTTA, Fernando e CALDAS, Miguel P. (org.). Cultura Organizacional e Cultura Brasileira. São Paulo: Atlas, 1997. p. 38-54.

HALL, Stuart. A identidade cultural na pós-modernidade. Rio de Janeiro: DP\&A, 1998.

HOFSTEDE, Geert. Cultures and organizations - software of the mind. New York: McGraw-Hill, 1991.

ISNARD, Ana L. Serviços de atendimento ao consumidor: a relação entre o posicionamento do SAC na empresa e a sua maior participação no processo decisório empresarial. In: XXI ENCONTRO NACIONAL DOS PROGRAMAS DE PÓS-GRADUAÇÃO EM ADMINISTRAÇÃO - ENANPAD. (1997: Rio de Janeiro). Anais ... Marketing. Rio de Janeiro: Associação Nacional de Programas de PósGraduação em Administração, 1997. p. 116. (Texto Integral em CD-ROM dos Anais do $21^{\circ}$ ENANPAD). 
JORNAL DO CONSELHO NACIONAL DE SAÚDE, ano I, n.1 Set./Out. 1998.

LARAIA, Roque de B. Cultura: um conceito antropológico. $11^{\mathrm{a}}$ ed. Rio de Janeiro: Jorge Zahar Editor, 1997.

MARTIN, Joanne. Cultures in organizations - three perspectives. Oxford: Oxford University Press, 1992.

MOOIJ, Marieke K. de. Global marketing and advertising: understanding cultural paradoxes. Thousand Oaks: Sage, 1998.

MORGAN, Gareth. Imagens da organização. São Paulo: Atlas, 1996.

MORRISON, Elisabeth W. e MILLIKEN, Frances J. Organizational silence: a barrier to change and development in a pluralistic world. The Academy of Management Review, v. 25, n. 4, p. 706-725, Out. 2000.

MYRDAL, Gunnar. O valor em teoria social. São Paulo: Pioneira e Editora da USP, 1965.

ORTIZ, Renato. Cultura brasileira e identidade nacional. $2^{\mathrm{a}}$ ed. São Paulo: Brasiliense, 1986.

PASTORE, Karina. O paraíso dos remédios falsificados. Veja, São Paulo, n. 1554, 8 jul. 1998. p. 4047.

PENTEADO FILHO, José R. W. Marketing no Brasil não é fácil. Rio de Janeiro: LTC, 1990.

PINSKY, Jaime e ELUF, Luiza N. Brasileiro(a) é assim mesmo: cidadania e preconceito. São Paulo: Contexto, 1993.

PRADO Jr., Caio. Formação do Brasil contemporâneo: colônia. 21 a ed. São Paulo: Brasiliense, 1989.

PRESTES MOTTA, Fernando C. Jeitinho brasileiro, controle social e competição. Revista de Administração de Empresas, São Paulo, v. 39, n.1, p. 6-12, Jan./Mar. 1999.

PRESTES MOTTA, Fernando C. e CALDAS, Miguel P. Introdução: cultura organizacional e cultura brasileira. In: PRESTES MOTTA, Fernando e CALDAS, Miguel P. (orgs.). Cultura Organizacional e Cultura Brasileira. São Paulo: Atlas, 1997. p. 15-21.

RIBEIRO, Darcy. O povo brasileiro: a formação e o sentido do Brasil. São Paulo: Companhia das Letras, 1995.

RICHERS, Raimar. Surfando as ondas do mercado. $2^{a}$ ed. São Paulo: RR\&CA Editora, 1996.

SANTOS, Cristiane P. dos. Reclamações de consumidores e respostas empresariais: uma abordagem inicial sobre o processo de marketing de defesa. In: XXI ENCONTRO NACIONAL DOS PROGRAMAS DE PÓS-GRADUAÇÃO EM ADMINISTRAÇÃO - ENANPAD. (1997: Rio de Janeiro). Anais ... Marketing. Rio de Janeiro: Associação Nacional de Programas de Pós-Graduação em Administração, 1997.p. 112-13. 
SCHWARTZ, Stuart. O país do presente. Veja, São Paulo, n. 1594, 21 abr. 1999. Entrevista.

SIQUEIRA, Márcia. Laboratório entra em rol de suspeitos. Estado de Minas Gerais, Belo Horizonte, 18 jul. 1998. p. 24.

SMIRCICH, Linda. Concepts of culture and organizational analysis. Administrative Science Quarterly, v. 28, n.3, p. 339-359, 1983.

SOUZA, Jessé. A ética protestante e a ideologia do atraso brasileiro. Revista Brasileira de Ciências Sociais, São Paulo, v. 13, n. 38, p. 97-116, Out. 1998. 